\title{
MRI Image Enhancement: Optimized Filtering Mechanism for Achieving High Accuracy in Diagnose Process
}

\author{
Abhinav Singh Andotra ${ }^{1}$ and Sandeep Sharma ${ }^{2}$ \\ Department of Computer Science, Guru Nanak Dev University, Amritsar, Punjab, India \\ E-Mail: abhinavthakur07@gmail.com
}

\begin{abstract}
Segmentation plays an important role in separating data from medicinal images and also helps in clinical findings. Segmentation is the way toward apportioning the image into different regions. MRI is utilized to extract images of delicate tissues of human body. It is utilized in analyzing the human organs without the requirement of surgery. For the most part MRI images contain a lot of noise caused by operator performance, equipment and the environment, which prompts genuine errors. MRI is a productive way in giving data in regards to the area of tumors and even the volume. The noise present in the MRI image can be evacuated by utilizing different de-noising procedures whichever is most appropriate method depending on the type of image obtained and afterward can be handled by any of the segmentation techniques. The noise in MRI images might be because of field strength, RF pulses, RF coil, voxel volume, or receiver bandwidth. In our proposed paper a review of different noise handling and filtering mechanism is conducted in order to enhance the quality of image. In this paper we modify the adaptive median filter by applying redundancy handling mechanism and enhance the contrast of image by applying histogram equivalence method.
\end{abstract}

Keywords: Noise handling, MSE, PSNR, Histogram equivalence, Adaptive median filter

\section{INTRODUCTION}

Medical field is greatly impacted with the problems present within capturing mechanism or transmission media. To tackle the issue, noise handling mechanisms such as filters are used. Filters however required modifications to tackle wide variety of noises In most of the techniques, noise handling is done but image becomes unstable and clarity is lost[1]. to tackle this issue, Gaussian smoothening and filtering is separately required to be applied on MRI image. A nonlinear strategy used to sustain the sharpness called the anisotropic dispersion filter has been proposed[2]. In this approach, pixel values are found the middle value from neighbourhoods, whose size and shape rely upon nearby image variety that is estimated at each point. The diffusion coefficient is chosen fromthe image slope so as to empowertranquiling inside a locale inclination to smoothing over the limits. Then again, the two-sided filter has been proposed in view of the change of the Gaussian filter. The technique suggested in [3] uses geometrical closeness to tackle the issue of noise handling. To determine the geometrical closeness, Euclidean distance can be applied. Distance obtained if is less than threshold then pixel is termed as correct. Otherwise pixel is added within corrupted list.
Noise is a fundamental challenge that is required to be tackled for medical diagnosis. Medical diagnosis process required to analyse characteristics of noises within MRI images.

\section{A. Noise Properties Common in MRI Images}

The primary source of noise within MRI images is thermal. Heat causes the free electrons within the image to be excited and when electron returns to ground state, extra charge is emitted causing noise within MRI images. Noise that appears due to heat causes white section within the image[4]. Such noise within the image is known as impulse noise. $\mathrm{K}$ mean clustering mechanism is used to locate the nearest pixels within the correct and incorrect clusters[5]. Correct clusters contain the pixel with correct in intensities hence image uniformity is preserved but if pixels also appear within incorrect clusters then uniformity is lost. This non uniformity is spread over the surface of the image causing distortion. This distortion is clearly visible on the image surface and difficult to rectify. Gaussian noise falls under this category. Shot noise also affects the clarity of the image and occurs due to environmental problems.

Diagnosis of medical images requires clarity. Clarity within accurate analysis of MRI images is imperative and hence existing filters requires some modification in terms of new noises and space conservation mechanism. Restoration of images to original contents is required and for that purpose peak signal to noise ratio and mean square error parameters requires optimizationRequirement of various noises tackling strategies from within MRI images including Rician noise is critical. This noise impact the credibility of information and wrong results could be extracted as a result. Image feature extraction and selection process could be direct or iterative in nature. Gaussian noise is another critical noise corrupting the image[7]. Smoothening operation is applied to the image to tackle the noise from within the image. Gaussian filter is specially created for this purpose which falls under the category of spatial filter. To handle such devastating noises, de-noising is an alternative to improve the picture clarity for more exact investigation[8]. The essential focus of picture de-noising systems is to clear such noises while holding however much as could sensibly be normal the imperative flag features. There are various manages the recovery of pictures corrupted by commotion. A couple of channels are used to oust commotion from a picture by making a 
confirmation of a more correct variation of pixels. By contemplating neighbouring pixels, over the top "tumultuous" pixels can be filtered through. Lamentably, unprecedented pixels can moreover address exceptional fine inconspicuous components, which can in like manner be lost in light of the smoothing strategy. There is no one of a kind method for noise expelling from influenced image[9]. Diverse calculations are utilized relying upon the noise display. The averaging filtering procedure can effectively expelnoise from the distorted image however for this circumstance the filtered image perseveres through the clouding sway. For the mean separating frameworks each pixel is considered to determine the mean and besides every pixel is supplanted by that figured mean. Henceforth points of interest and edges are not recouped attractively, particularly when the noise level is high[10][11]. This paper looks at basic noise evacuating calculations and presents another calculation for noise lessening from restorative images that uses adaptive median filter technique and histogram equivalence contrast enhancement method. The test result demonstrates the adequacy of the proposed technique.

\section{B. Statistical Parameters Utilized for Analyzing the De- noised Image}

There are various methods of estimation are used for examination of the image that are to be executed[12]. The execution improvement can be accessed using signal tonoise ratio (SNR), mean square error (MSE) and peaksignal to-noise ratio (PSNR). How the signal has been corrupted by various types of noise can be measured usingSignal tonoise proportion (SNR). The various distinctions of the pixels in an image can be measured using mean square error (MSE) and also it averages the sum of contrast between two images. The proportion between energy consumed by the signal and energy of noise that impact the representation[13], is the PSNR (Peak signal to noise ratio).

\section{Adaptive Median Filter}

The fundamental median filter is the standard adaptive median filter. In this technique, a square window of size $2 \mathrm{k}+1$, where $\mathrm{k}$ goes from 1 to $\mathrm{N}$, is utilized to channel the focus pixel. The pixels in the window are first arranged and the inside pixel is changed to the middle estimation of the arranged grouping. This strategy is the most straightforward of the middle filtering systems and in light of its effortlessness; it has been utilized for quite a while[14]. The second kind of middle filtering is the inside weighted middle filtering strategy. This is like the standard middle filtering system with the exception of that the inside pixel in the window to be separated is appointed a specific weight, i.e. the inside pixel is rehashed for some number of times which is characterized as the weight. Since the middle pixel in the window to be separated is rehashed for some number of times, this system is known as the inside weighted adaptive median filter[15].

\section{Histogram Equivalence}

Histogram equalization is a method to process images in order to adjust the contrast of an image by modifying the intensity distribution of the histogram. The objective of this technique is to give a linear trend to the cumulative probability function associated to the image.The processing of histogram equalization relies on the use of the cumulative probability function (cdf).

\section{RELATED WORKS}

In this paper, it is proposed that a hybrid approach is used to reduce noise in MRI images and enhance images[16]. It utilizes Anisotropic Diffusion Filter with modified Decision Based Un symmetric Trimmed median filter to remove noise from the images that reduce their quality. Also this technique preserves the details and edges are enhanced in MRI image. The results evaluated proved that is better than the technique utilized earlier. The performance of the proposed system is measured using various parameters and also it enhances quality, preserve edge details and remove noise.

In this paper, it is proposed that a method called adaptive median filter and also compared it with mean filter and median filter[17]. This technique is utilized to remove noise from MRI images. It removes speckle noise of the medical images. The experiment shows that the proposed methodology is better than any other. But it must be enhance further as de-noising of medical image is very challenging task.

This paper explores the execution assessment of three diverse image de-noising methods utilized to refine poor tumor limits in complex MRI brain tumor images corrupted by Rician noise[18]. Denosing comes about demonstrate that image quality enhances with a mix of Gauss filter and $\mathrm{ADF}$ filter. It can be seen that traditional division systems alone would not bring better outcomes. Further precision of tumor is observed to be enhanced utilizing these systems. Extraction comes about uncover that Bayesian noise and Poisson noise strategies joined with area developing would give acceptable outcomes in less time contrasted with alpha noise. Also for complex images the Poisson noise can be handled by using region growing technique.

In this paper, it is proposed that a new technique to reduce noise in MRI images named linear minimum mean square error (LMMSE)[19]. This technique automatically estimates the noise power of an MRI image utilizing a set of methods. These functions utilized statistical distribution of an image and according to them it estimates the noise dynamically. It clear noise in images and preserve various feature that are extracted from images. It utilizes statistical measures like local variance, mean deviation, standard deviation and also the derivation can be shown using Rayleigh model. 
This paper explores that the technique named hybrid Cmeans clustering for enhancing MRI images and segmenting[20]. It gives hybridization of fuzzy $c$ mean clustering and modified filtering mechanism for denoising and classification of images. The results verify that it is good and also slow the execution time.

It combines the Susceptibility-Weighted Imaging (SWI) with magnitude and phase information that gives high resolution MRI images[21]. It utilizes denoising filter that preserve small structures and enhance the image quality. It introduce another committed noise expulsion calculation in light of the Non-Local Means (NLM) filter and contrast its outcomes and the first SWI and "standard" NLM-denoised MRI images. Both the visual appraisal by two master per users and the quantitative assessment of the difference changes of the voxel powers exhibited that the images reestablished with the proposed calculation fared reliably superior to anything the other two plans, demonstrating that a legitimate treatment of noise in the complicated MRI dataset may prompt upgrades of the general SWI quality.

\section{PROPOSED METHODOLOGY}

The proposed system takes into consideration all the noises. The filter which we are proposing is adaptive median filter which takes into consideration both the noise and rectify and restore the images in efficient manner.

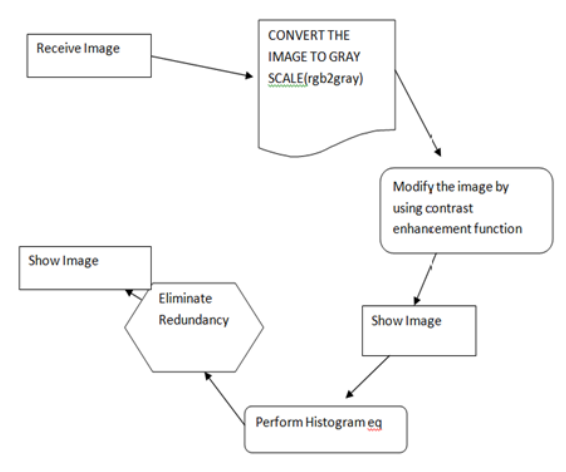

Fig. 1 Proposed methodology

The proposed method is used in order to overcome the problems in existing papers.

1. Input Image

2. I=inread(image)

3. Apply enhanced adaptive median filter

Buffer=I(x,y)

4. If (Buffer $i==i(x, y)$ )

Discard Pixel

End of if

5. I=Adaptive (Buffer)

6. Apply Histogram Equalisation technique to enhance the colour contrast

At the end Output comes in the form of PSNR and MSE.
MSE: Mean square Error is used to estimate unobserved quantities by measuring the errors average with squares. In other words the distinction of estimated error and the actual error is MSE. The function of MSE consists of the squared error loss or quadratic loss's expected values. The formula to compute the MSE is given below:

$$
M S E=\frac{1}{m n} \sum_{i=0}^{m-1} \sum_{j=0}^{n-1}[I(i, j)-K(i, j)]^{2}
$$

PSNR: The proportion between energy consumed by the signal and energy of noise that impact the representation, is the PSNR (Peak signal to noise ratio). PSNR can be easily computed by the use of MSE. The formula to compute the PSNR is given below:

$$
\begin{aligned}
P S N R & =10 \cdot \log _{10}\left(\frac{M A X_{I}^{2}}{M S E}\right) \\
& =20 \cdot \log _{10}\left(\frac{M A X_{I}}{\sqrt{M S E}}\right) \\
& =20 \cdot \log _{10}\left(M A X_{I}\right)-10 \cdot \log _{10}(M S E)
\end{aligned}
$$

Here MAXi is the highest pixel value of image.

\section{FLOWCHART}

The flow diagram showing the working of Adaptive median filter is described as under:

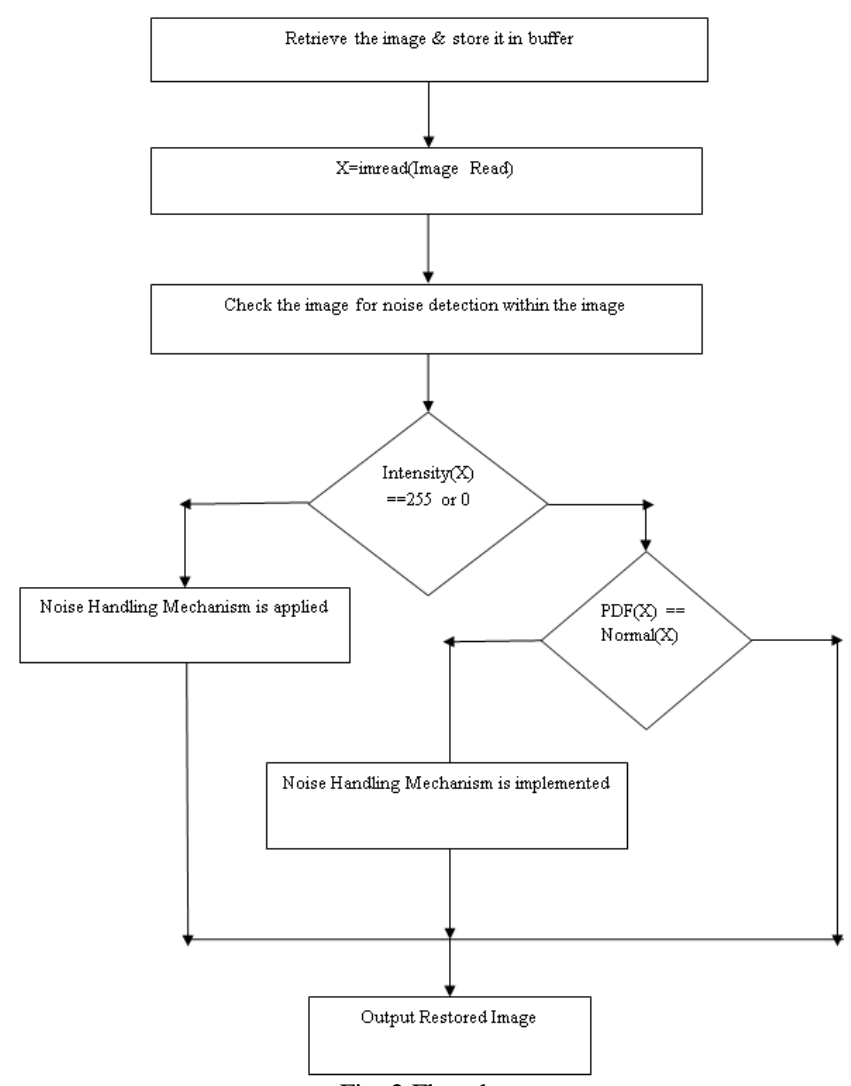

Fig. 2 Flowchart 


\section{RESULTS}

The simulation of the adaptive median filter is accomplished within the MATLAB tool. The MATLAB is a mathematical tool in which digital image processing can be handled efficiently. The Simulation results shows that clarity of the image is introduced using the proposed mechanism.
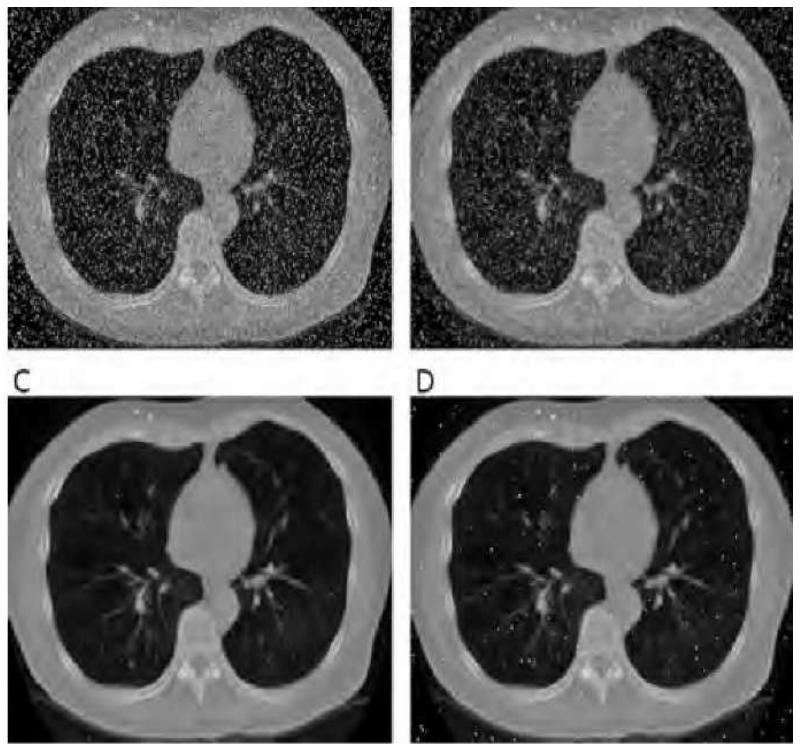

Fig. 3 Restoration of image from impulse \& gaussian noise

The first image is corrupted with impulse and Gaussian noise. When the adaptive median filter is applied the image is improved by eliminating salt and pepper noise from the image. In the next phase Gaussian noise is also eliminated and image is enhanced.

\section{A. Parameter-MSE}

TABLE I DEGREE OF RELATIONSHIP B/W THE PIXEL IN TERMS OF MEAN SQUARE ERROR

\begin{tabular}{|c|c|c|}
\hline SIMULATION & MSE EXISTING & MSE PROPOSED \\
\hline SIM 1 & 15.067 & 6.167 \\
\hline SIM 2 & 15.789 & 6.189 \\
\hline SIM 3 & 14.567 & 5.167 \\
\hline SIM 4 & 14.892 & 5.898 \\
\hline SIM 5 & 14.557 & 5.557 \\
\hline SIM 6 & 15.087 & 6.087 \\
\hline SIM 7 & 15.769 & 6.769 \\
\hline SIM 8 & 14.527 & 5.527 \\
\hline SIM 9 & 14.110 & 5.110 \\
\hline SIM 10 & 14.235 & 5.235 \\
\hline
\end{tabular}

The MSE will indicate the degree of relationship between the pixels. The existing system has moreMSE associated with it.

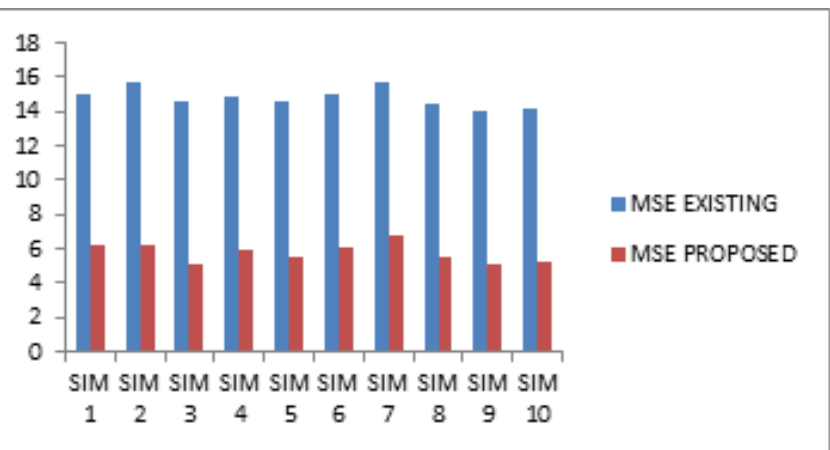

Fig. 4 Proposed mse and existing mse

The simulation result indicates that the proposed system is better as compared to existing system.

\section{B. Parameter-PSNR}

The PSNR of the proposed system is more as compared to the existing system

TABLE II EXISTING SYSTEM HAS LESS PSNR AS COMPARED TO PROPOSED SYSTEM

\begin{tabular}{|c|c|c|}
\hline SIMULATION & PSNR Existing & PSNR Proposed \\
\hline SIM 1 & 21 & 62.23 \\
\hline SIM 2 & 24.26 & 65.212 \\
\hline SIM 3 & 20.256 & 66.235 \\
\hline SIM 4 & 21.235 & 65.235 \\
\hline SIM 5 & 35.235 & 52.235 \\
\hline SIM 6 & 32.789 & 56.235 \\
\hline SIM 7 & 30.231 & 50.235 \\
\hline SIM 8 & 35.2646 & 60.24 \\
\hline SIM 9 & 34.265 & 56.235 \\
\hline SIM 10 & 34.2556 & 50.256 \\
\hline
\end{tabular}

The tabular representation shows that existing system has lessPSNR as compared to proposed system. The chart is as follows:

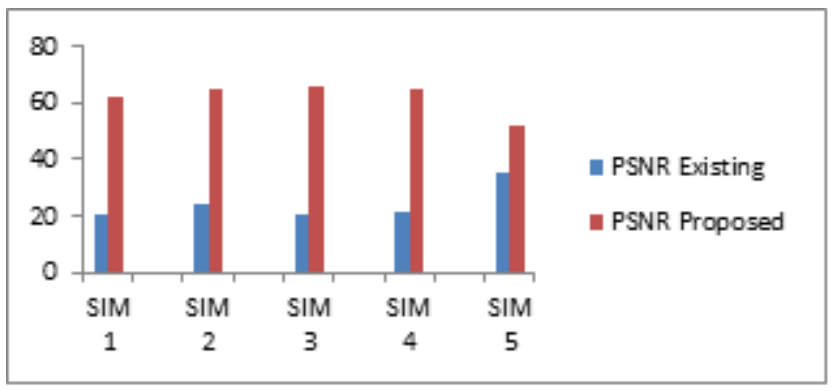

Fig. 5 PSNR in existing and proposed system

The simulation indicates that proposed system is better as compared to existing system. 


\section{Parameter-Accuracy}

The accuracy of the proposed system is better as compared to the existing system. The tabular representation of the existing and proposed system is as follows:

TABLE III PARAMETER ACCURACY IN PROPOSED AND EXISTING SYSTEM

\begin{tabular}{|c|c|c|}
\hline SIMULATION & Accuracy Existing & Accuracy Proposed \\
\hline SIM 1 & 84.562 & 96.546 \\
\hline SIM 2 & 89.562 & 98.564 \\
\hline SIM 3 & 85.656 & 95.265 \\
\hline SIM 4 & 85.213 & 96.36 \\
\hline SIM 5 & 84.56 & 96.256 \\
\hline SIM 6 & 82.565 & 95.2365 \\
\hline SIM 7 & 85.265 & 90.256 \\
\hline SIM 8 & 86.325 & 96.3256 \\
\hline SIM 9 & 87.2565 & 97.2568 \\
\hline SIM 10 & 88.2655 & 98.2352 \\
\hline
\end{tabular}

The accuracy associated with the proposed system is better as compared to existing system.

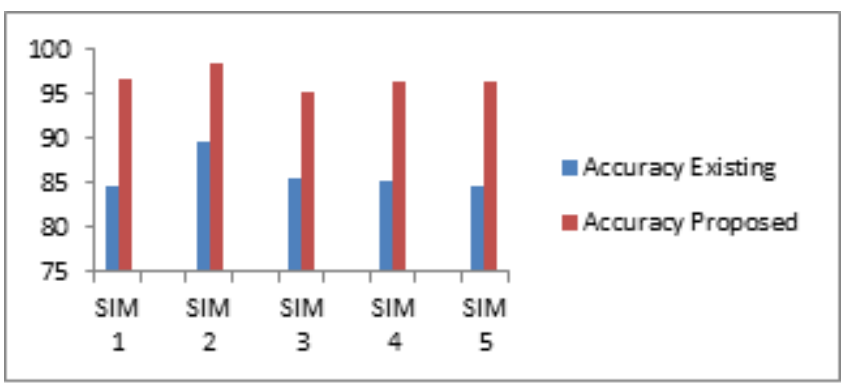

Fig. 6 Speed comparison between existing and proposed system

The simulation through the chart indicates that accuracy of the proposed system is better as compared to the existing system.

\section{CONCLUSION}

The proposed model combines the use of adaptive filters and histogram equivalence method. The technique can be used in order to eliminate the noises like salt and pepper and Gaussian noise from within the image. The filter produced better result as compared to individual use of median and homomorphic filter. The adaptive filter will automatically identify the noise and apply the noise handling mechanism accordingly. In case both the noises are corrupting the image then both noises are automatically handled in the present situation.

In the future we try to accommodate more noise handling mechanisms within the adaptive median filter so that single filter can be used to tackle all the noises present within the image.

\section{REFERENCES}

[1] G. Deng and L. W. Cahill, “Guassian filter for noise reduction,” in 1993 IEEE Conference Record Nuclear Science Symposium and Medical Imaging Conference, pp. 1615-1619.

[2] M. Kaur and R. Kaur, "Review of various filters for noise reduction,” pp. 238-242, 2016.

[3] Y. Ma, D. Lin, B. Zhang, Q. Liu, and J. Gu, “ A PCNN Time matrix used for noise removal,” in 2007 IEEE International Conference on Signal Processing and Communications, pp. 1499-1502, 2007.

[4] N. Vyas, A. Jain, and C. P. Singh, "A Review of high density function for noise removal,” pp. 1613-1616, 2016.

[5] P. Singh, "Review of image restoration techniques," IEEE ACCESS, Vol. 149, No. 1, pp. 18-27, 2016.

[6] L. Liang, J. Wang, J. Song, and A. S. Model, “ an algorithm for noise removal using CNGSS," no. 5, pp. 1-3.

[7] A. Kandpal and V. Ramola, "Image segmentation using various filters,” Vol. 3, No. 3, pp. 201-206, 2015.

[8] G. Wang, D. Li, W. Pan, and Z. Zang, "Noise removal using Modified switching median filter," Signal Processing, Vol. 90, No. 12, pp. 3213-3218, 2010.

[9] M. P. Mahajan, E. Engineering, and E. Engineering, “ Review of various noise removal filter utilized non linear methodology,” pp. 6820-6828.

[10] A. June, “An algorithm for noise reduction based on Fuzzy system,” Vol. 2, No. 2, 2014.

[11] A. Singh, " Survey of various noise removal techniques for removing impulse noise,” IEEE ACCESS, Vol. 3, No. 6, pp. 659-665, 2014.

[12] E. E. Kerre, D. Van De Ville, M. Nachtegael, D. Van Der Weken, and E. E. Kerre, “ By Using fuzzy image filtering noise removal . IEEE,” Vol. 125050, No. January 2013, 2003.

[13] R. Pandey, A. Awasthi, and V. Srivastava, " the comparative study of Bit error rate and SNR in OFDM,” Vol. 2013, No. Cac2s, pp. 463466, 2013.

[14] A. Agrawal, A. Choubey, and K. K. Nagwanshi, "Noise Reduction in medical image using adaptive fuzzy based Image Filtering techniques,” Vol. 2, No. 4, pp. 1457-1461, 2011.

[15] S. H. Teoh and H. Ibrahim, "Analysis of Median Filtering Frameworks : A Literature Survey,” Vol. 1, No. 4, pp. 4-7, 2012.

[16] U. R and P. K, "Hybrid Approach for Noise Removal and Image Enhancement of Brain Tumors in Magnetic Resonance Images," Adv. Comput. An Int. J., Vol. 7, No. 1/2, pp. 67-77, 2016.

[17] A. S. Y. Bin-habtoor and S. S. Al-amri, "Removal Speckle Noise from Medical Image Using Image Processing Techniques,” Int. J. Comput. Sci. Inf. Technol., Vol. 7, No. 1, pp. 375-377, 2016.

[18] S. Renukalatha, "Brain Tumor Analysis of Rician Noise Affected MRI Images,” vol. 141, no. 14, pp. 26-33, 2016.

[19] S. Aja-Fernández, C. Alberola-López, and C. F. Westin, "Noise and signal estimation in magnitude MRI and Rician distributed images: A LMMSE approach,” IEEE Trans. Image Process., Vol. 17, No. 8, pp. 1383-1398, 2008.

[20] A. Srivastava, A. Asati, and M. Bhattacharya, “A Fast and NoiseAdaptive Rough-Fuzzy Hybrid Algorith for Medical Image Segmentation,” IEEE Int. Onference Bioinforma. Biomed., pp. 416421, 2010.

[21] P. Borrelli, E. Tedeschi, S. Cocozza, C. Russo, M. Salvatore, G. Palma, M. Comerci, B. Alfano, and E. M. Haacke, “ NLM -based noise removal technique for Improving SNR,” IST 2014 - 2014 IEEE Int. Conf. Imaging Syst. Tech. Proc., Vol. 2, No. 3, pp. 346-350, 2014. 